\title{
3D MODELLING OF THE MAMARI TABLET FROM THE RONGORONGO CORPUS: ACQUISITION, PROCESSING ISSUES, AND OUTCOMES
}

\author{
L. Lastilla ${ }^{\mathrm{a}, \mathrm{b}}{ }^{*}$ R. Ravanelli ${ }^{\mathrm{c}}$, M. Valério ${ }^{\mathrm{c}}$, S. Ferrara ${ }^{\mathrm{c}}$ \\ ${ }^{a}$ Department of Computer, Control and Management Engineering Antonio Ruberti (DIAG) - Sapienza University of Rome, Italy \\ ${ }^{\mathrm{b}}$ Sapienza School for Advanced Studies, Rome, Italy \\ ${ }^{c}$ Department of Classical Philology and Italian Studies (FICLIT), Alma Mater Studiorum - University of Bologna, Italy \\ lorenzo.lastilla@uniroma1.it \\ $<$ r.ravanelli, miguel.valerio, s.ferrara $>@$ unibo.it
}

Technical Commission II

KEY WORDS: 3D modelling, Laser scanning, Structured light, Precise 3D digitization, Rongorongo inscriptions

\begin{abstract}
:
Rongorongo is an undeciphered script inscribed on wooden objects from Easter Island (Rapa Nui) in the Pacific Ocean. The existing editions of the inscriptions, and their widespread locations in museums and archives all over the world today constitute a serious obstacle to any objective paleographical assessment. Thus, with a view to a potential decipherment, creating 3D models of the available corpus is of crucial importance, and one of the objectives of the ERC INSCRIBE project, based at the University of Bologna with Professor S. Ferrara as Principal Investigator. In this preliminary work, we present the results of the 3D digitization of the Mamari tablet, one of the longest inscriptions in Rongorongo, housed in the Museum Archives of the Congregazione dei Sacri Cuori di Gesù e Maria in Rome. The tablet is made of wood, with a shiny reflecting surface, characterized by a mainly dark texture. The 3D modelling was carried out with the ScanRider 1.2 laser scanner manufactured by VGER, based on Structured Light technology, taking care to ensure the legibility of each sign while preserving the overall shape of the object as precisely as possible. To overcome the difficulties inherent in the object's complex fabric, the Mamari tablet was acquired in separate sections (joined together during processing through specific markers), thus managing to optimize the optical parameters of the laser scanner, such as the exposure of the camera and the depth of field of the projector. Furthermore, an evaluation of the 3D reconstruction precision was also carried out, highlighting a precision of few hundredths of millimeters, in agreement with the claimed nominal standard deviation. In addition to the 3D model produced, one of the main results of this endeavor was the definition of a successful method to scan such complex objects, which will be replicated to finalize the complete 3D modelling of the whole Rongorongo corpus of inscriptions.
\end{abstract}

\section{INTRODUCTION}

Rongorongo is an undeciphered script of Easter Island or Rapa Nui (Chile), whose corpus is preserved on twenty-six inscribed wooden objects, each one identified by a single-letter code in Thomas Barthel's nomenclature system (Barthel, 1958).

Even if among the scholars there is still disagreement on the nature of the Rongorongo writing system, several attempts of decipherment have been made over the years. The great interest surrounding this writing system of Easter Island is due to its possible creation "from scratch": indeed, the Rongorongo script could be one of the few original inventions of writing in the history of mankind.

The widespread distribution of Rongorongo inscriptions in museums and institutions all over the world is one of the main obstacles to their analysis, as the limited number of inscriptions hinders any attempt at decipherment. This makes it difficult to analyze the inscriptions in terms of paleography, especially as their editions do not always agree (Barthel, 1958; Fischer, 1997 apud Spaelti, 2005-2019). Thus, especially with a view towards potential decipherment, the $3 \mathrm{D}$ modelling of the available corpus would be of crucial importance. It would provide scholars with more accurate sources and allow them to reassess the existing handmade line-drawings: this is why the Rongorongo digitization process is one of the objectives of the ERC INSCRIBE -

\footnotetext{
${ }^{*}$ Corresponding author.
}

'INvention of SCRIpts and their BEginnings' - project, based at the University of Bologna with Professor Silvia Ferrara as Principal Investigator (INSCRIBE, 2018).

In this preliminary work, we present the result of the 3D digitization of Text $\mathrm{C}$ or Mamari, one of the longest inscriptions in Rongorongo (consisting of 1000 glyphs distributed along 28 lines), housed in the Museum Archives of the Congregazione dei Sacri Cuori di Gesù e Maria in Rome. The Mamari tablet is particularly relevant because it contains the only Rongorongo inscription whose interpretation is compelling and consensual. Namely, its recto contains four lines of text representing a calendar for the lunar month, whose signs are arranged in a scheme that squares well with the Rapa Nui lunar month, as documented from oral sources (Barthel, 1958; Guy, 1990).

Apart from presenting the digitization methodology and procedure (Section 3) and the 3D model produced, this contribution includes also an evaluation of the $3 \mathrm{D}$ reconstruction precision (Section 4.1) and showcases several shaders and filters available on the freely available software CuneiformAnalyser (Fisseler et al., 2013) to increase the sign legibility and thus to allow for a more complete visual and automatic inspection of the object's 3D model (Section 4.2).

\section{CASE STUDY}

The Mamari (shown in Figure 1) is a wooden tablet which was collected on Easter Island, inscribed with signs of the Rongorongo 


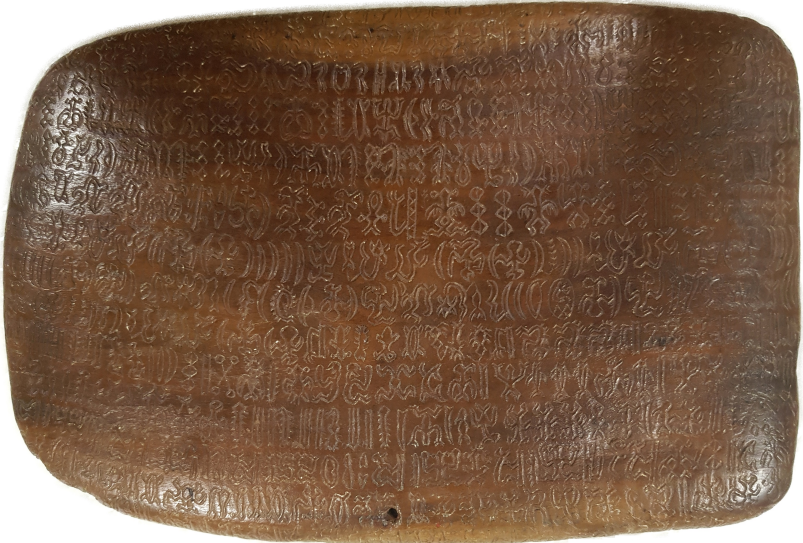

Figure 1: Mamari tablet (side a). Courtesy of Museum Archives of the Congregazione dei Sacri Cuori di Gesù e Maria in Rome

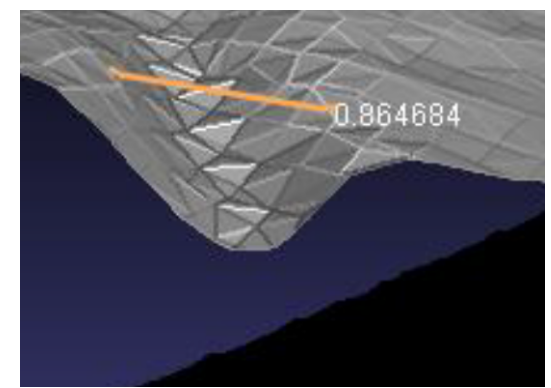

Figure 2: Detail of a sign in the 3D model produced (the scale is expressed in millimeters)

script. This object has a rectangular shape, whose size measures $29 \mathrm{~cm} \times 19.6 \mathrm{~cm} \times 2.5 \mathrm{~cm}$ (Fischer, 1997). The wood of the tablet - Thespesia Populnea or Pacific rosewood according to (Orliac, 2005) - is highly reflective and has a mainly dark texture. Moreover, the glyphs are not deeply incised if we compare their depth, which amounts to approximately $0.2 \mathrm{~mm}$, with the thickness of the object (see the detail of a sign in Figure 2).

Furthermore, an important aspect to consider when trying to reconstruct the 3D geometry of the tablet is that it essentially has two prevailing dimensions: because this study is functional to reconstruct its overall 3D shape, and not to produce 2.5D models, the edges have to be acquired with particular care.

All these features make it challenging to reconstruct the 3D geometry of the tablet with a standard photogrammetric approach. For this reason, the 3D modelling was carried out with the ScanRider 1.2 laser scanner manufactured by VGER, based on Structured Light technology. ScanRider 1.2 was chosen due to its high performances in terms of nominal precision, accuracy and resolution required to reconstruct the smallest details or high frequencies of the object, such as the inscriptions themselves (Lastilla et al., 2019; Affatato et al., 2017).

\section{METHODOLOGY, EXPERIMENTAL SETUP, DATA ACQUISITION AND PROCESSING}

ScanRider 1.2 by VGER is a high-resolution laser scanner based on Structured Light technology. To apply the Structured Light principle, ScanRider 1.2 is endowed with a digital light processing projector and a panchromatic (black and white) camera: the object, placed on a turntable, is therefore acquired in all of its sides. The scanner does not have its color camera, thus it cannot provide texture to the $3 \mathrm{D}$ models.

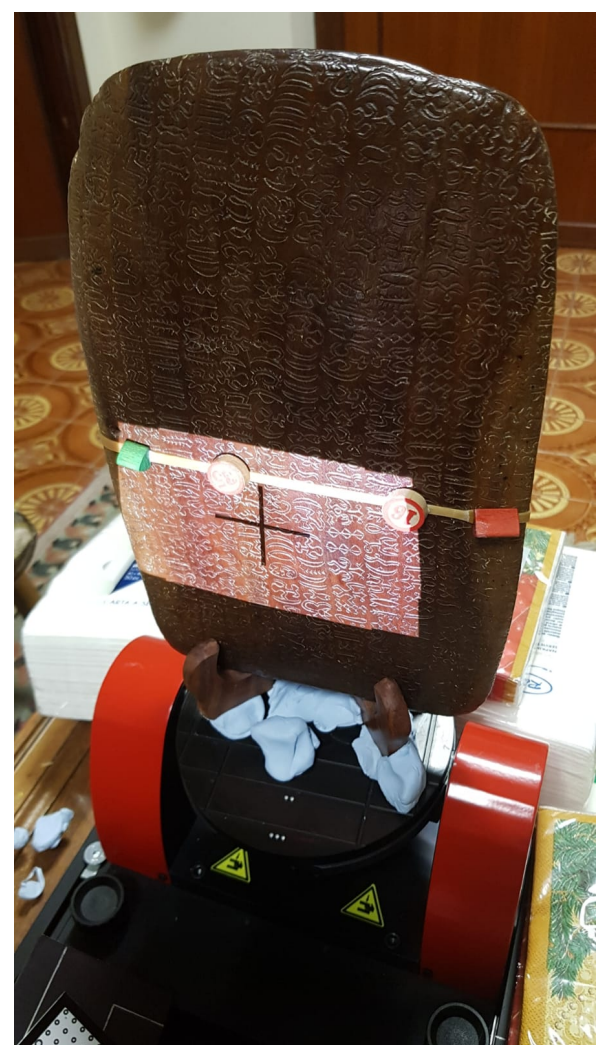

Figure 3: Markers; the illuminated area corresponds to the surface acquired in a single scan carried out in area 2

The scanning process follows an initial stage of calibration: during this step, the camera and the projector parameters are estimated and a 3D reference frame is defined. ScanRider 1.2 has three different scanning volumes: the smaller the volume, the more precise and accurate the model (Lastilla et al., 2019). To find a compromise between global accuracy - thus preserving the overall shape of the object as precisely as possible - and local one (with the aim of ensuring the legibility of each sign) and to avoid the acquisition of an excessive amount of data, the intermediate scanning volume (nominal standard deviation $\leq 0.07 \mathrm{~mm}$ ) was used.

To overcome the difficulties inherent in the object's complex fabric, the Mamari tablet was acquired in separate sections, joined together during the processing step through specific markers made of small pieces of wood - thus having optical characteristics similar to those of the tablet - placed on elastic bands (Figure 3). In this way the tablet surface was divided into five sub-areas (Figure 4), each one captured at least twice, one with two elastic bands and one with only one, to increase the redundancy and, at the same time, to reconstruct also the signs placed below the markers. On the one hand, this acquisition strategy allowed to optimize the optical parameters of the laser scanner - such as the exposure of the camera and the depth of field of the projector - for each considered portion of the tablet, leading to a more accurate reconstruction of the signs. On the other, though, this methodology lengthened the duration of the scanning process, not taking advantage of the automatic procedure the scanner offers.

The alignment of the scans was carried out within the dedicated SpaceRider software. Considering that the tablet edges and signs are too thin to guide the process, the alignment relied on the 3D geometry of the markers. After the alignment, the markers were removed and the scans were fused and smoothed, obtaining the final 3D model. 


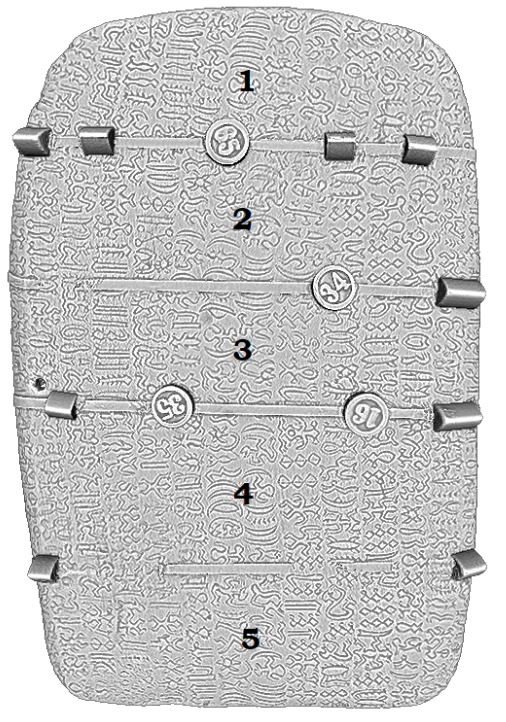

Figure 4: The markers on the 3D model and the five sub-areas

The whole processing was carried out on a Dell XPS 15 notebook, having a $5 \mathrm{GHz} 8$-Core Intel i9-9980HK processor, a 32 GB $2.666 \mathrm{MHz}$ DDR4 memory and a NVIDIA GeForce GTX 1650 graphics card.

\section{RESULTS}

Overall, 1090 scans were acquired, for a total of about five and a half million points and more than one billion triangles. The raw scans were thus aligned, cleaned from the markers, fused and smoothed, producing the 3D model of the Mamari tablet (Figure 5) (INSCRIBE, 2019), characterized by about four million points and eight million triangles.

\subsection{Precision evaluation}

After the 3D model generation was completed, a precision evaluation of the model itself was carried out: this was possible because each sub-area which the tablet was divided into was acquired at least twice (more precisely, each sub-area was reconstructed using at least two groups of multiple scans). On the other hand, the accuracy of the 3D model was not estimated, since at present a more accurate $3 \mathrm{D}$ model of the tablet does not exist to be used as reference for comparison.

To evaluate the precision of the 3D model produced, a local procedure was followed: first, three well-distributed areas (visible in Figure 5) were selected from the Mamari 3D model. Thereafter, for each selected area, the pairwise distance between fused scan groups including that area was computed. In particular, for each couple of groups a point-to-mesh distance was computed through the Cloud/Mesh distance tool on CloudCompare software (Girardeau-Montaut, 2019).

For the area selected on side a (referred to as 1 in Figure 5), four fused scan groups were taken into account: one of them was chosen as reference and the distance between this reference and each one of the other three groups was computed on CloudCompare. The point-to-mesh signed distances obtained this way underwent the following statistical analysis: first of all, to exclude possible outliers or points external to the overlap area among all the four groups, the distance values out of the range $(-0.1 \mathrm{~mm}, 0.1 \mathrm{~mm})$ were removed. Hence, several statistical indicators were computed over for the obtained distances.
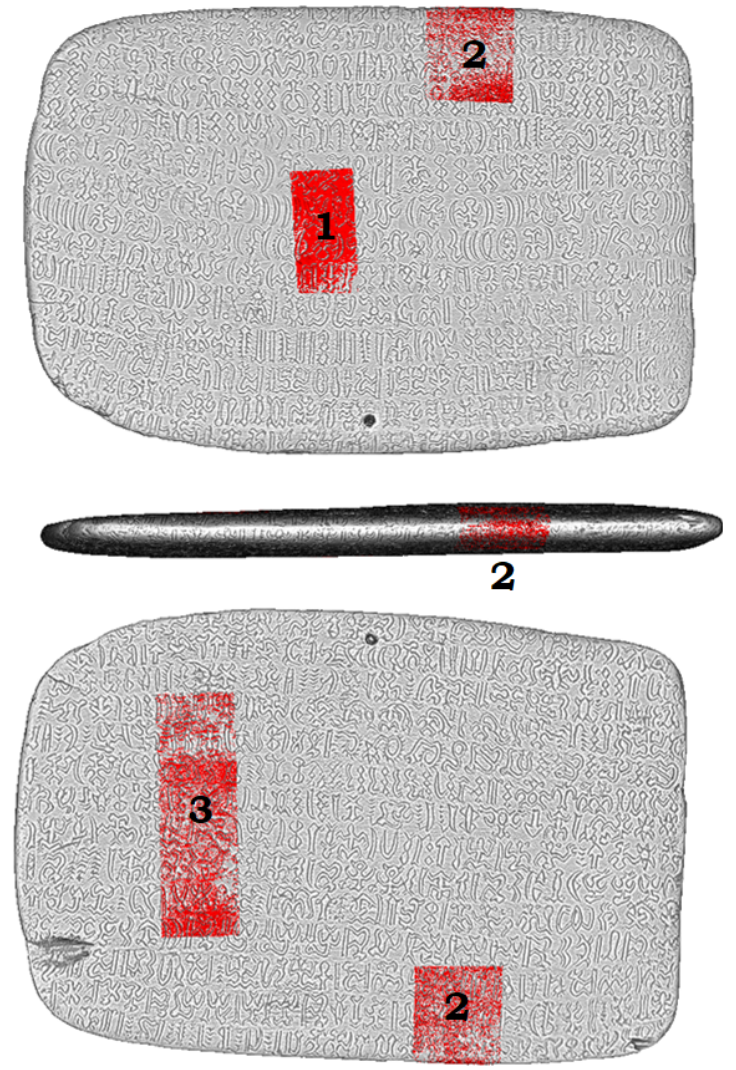

Figure 5: 3D model of the Mamari tablet, visualized through the Radiance Scaling shader implemented in CuneiformAnalyser software framework; in red the selected areas for precision evaluation

The same procedure as area 1 was repeated for the area selected on the border (area 2 in Figure 5) and for the one selected on side $\mathrm{b}$ of the Mamari tablet (area 3 in Figure 5). However, this time three scan groups were used instead of four. The statistical indicators obtained for the three areas are summarized in Table 1, Table 2 and Table 3. The results are very homogeneous over the three areas and highlight a mean precision of about three-hundredths of $\mathrm{mm}$, in agreement with the claimed nominal standard deviation. Finally, the slightly higher values of the standard deviation compared to NMAD show the permanence of some outliers in the samples. In Figure 6, an example of a distance histogram for group 3 of side a is given: it is worth noticing that the distance distribution can be considered approximately normal, and that this conclusion is true for all the groups included in the statistical analysis.

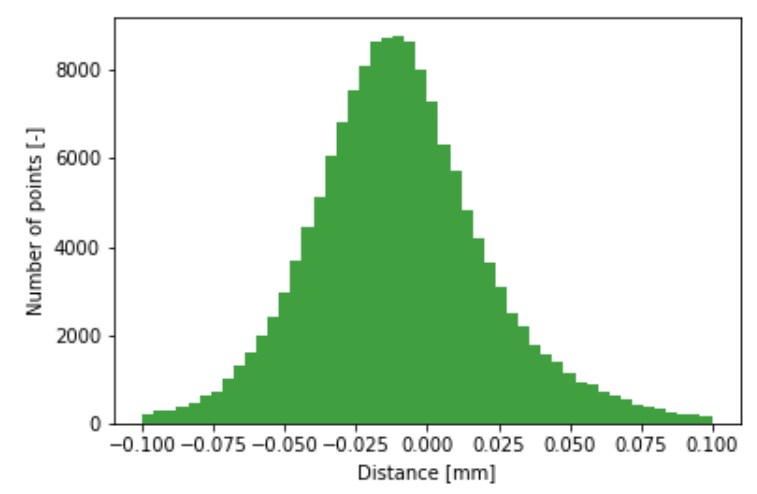

Figure 6: Example of distance histogram for group 3 of side a 


\begin{tabular}{ccccccccc}
\multirow{2}{*}{ Group } & mean & std. dev. & RMSE & median & NMAD & LE68 & LE95 & Number of points \\
\cline { 2 - 8 } & {$[\mathrm{mm}]$} & {$[\mathrm{mm}]$} & {$[\mathrm{mm}]$} & {$[\mathrm{mm}]$} & {$[\mathrm{mm}]$} & {$[\mathrm{mm}]$} & {$[\mathrm{mm}]$} & {$[-]$} \\
\hline 1 & -0.014 & 0.036 & 0.039 & -0.017 & 0.030 & 0.033 & 0.077 & 165227 \\
\hline 2 & -0.015 & 0.034 & 0.037 & -0.018 & 0.032 & 0.033 & 0.070 & 147338 \\
\hline 3 & -0.010 & 0.031 & 0.033 & -0.011 & 0.028 & 0.029 & 0.065 & 150342 \\
\hline mean & -0.013 & 0.034 & 0.036 & -0.016 & 0.030 & 0.032 & 0.070 & 154302 \\
\hline
\end{tabular}

Table 1: Statistical indicators computed for area 1 (side a)

\begin{tabular}{ccccccccc}
\multirow{2}{*}{ Group } & mean & std. dev. & RMSE & median & NMAD & LE68 & LE95 & Number of points \\
\cline { 2 - 8 } & {$[\mathrm{mm}]$} & {$[\mathrm{mm}]$} & {$[\mathrm{mm}]$} & {$[\mathrm{mm}]$} & {$[\mathrm{mm}]$} & {$[\mathrm{mm}]$} & {$[\mathrm{mm}]$} & {$[-]$} \\
\hline 1 & 0.014 & 0.032 & 0.035 & 0.013 & 0.030 & 0.031 & 0.064 & 1413130 \\
\hline 2 & 0.012 & 0.030 & 0.032 & 0.011 & 0.028 & 0.029 & 0.061 & 1463943 \\
\hline mean & 0.013 & 0.031 & 0.033 & 0.012 & 0.029 & 0.030 & 0.063 & 1438537 \\
\hline
\end{tabular}

Table 2: Statistical indicators computed for area 2 (border)

\begin{tabular}{ccccccccc}
\multirow{2}{*}{ Group } & mean & std. dev. & RMSE & median & NMAD & LE68 & LE95 & Number of points \\
\cline { 2 - 8 } & {$[\mathrm{mm}]$} & {$[\mathrm{mm}]$} & {$[\mathrm{mm}]$} & {$[\mathrm{mm}]$} & {$[\mathrm{mm}]$} & {$[\mathrm{mm}]$} & {$[\mathrm{mm}]$} & {$[-]$} \\
\hline 1 & 0.007 & 0.030 & 0.031 & 0.007 & 0.027 & 0.027 & 0.063 & 453567 \\
\hline 2 & 0.024 & 0.031 & 0.039 & 0.022 & 0.030 & 0.030 & 0.064 & 471859 \\
\hline mean & 0.015 & 0.031 & 0.035 & 0.015 & 0.028 & 0.029 & 0.064 & 462713 \\
\hline
\end{tabular}

Table 3: Statistical indicators computed for area 3 (side b)

\subsection{Visual enhancement of 3D surface features and sign leg- ibility}

For the very purpose of increasing the sign legibility and allowing for a more complete inspection of the inscribed tablet, the 3D model of the Mamari was rendered through CuneiformAnalyser software framework (Fisseler et al., 2013), which allows a unified and effective three-dimensional approach of analyzing and reconstructing manuscripts in the field of philological research.

CuneiformAnalyser implements indeed advanced rendering techniques using ambient occlusion data, Radiance Scaling (Vergne et al., 2010) - a shader which allows depicting shape through shading via the modification of light intensities around specific features like concavities and convexities - and curvature enhancement (with different options, such as Gaussian curvature, maximum curvature or minimum one) for a dynamic detail improvement and a realistic visualization of the 3D model (Fisseler et al., 2013). Moreover, the same software allows for assigning the mesh several materials (such as clay, gold, steel, etc.) and for adjusting the reflectance properties of the selected material, which also contributes to the enhancement of sign legibility (INSCRIBE, 2019). Thus, the use of these shaders allowed to visually evaluate the reconstruction of the signs (Figure 7).

\section{CONCLUSIONS AND FUTURE PROSPECTS}

In this paper, we showcase the procedure implemented to acquire the first ever 3D model of a Rongorongo inscription, Text $\mathrm{C}$ or Mamari tablet. In addition to the 3D model, one of the main results of this endeavor was the definition of a successful method for reconstructing the $3 \mathrm{D}$ geometry of a complex object like the Mamari tablet, which will be replicated to finalize the complete $3 \mathrm{D}$ modelling of the whole Rongorongo corpus of inscriptions.

Moreover, an evaluation of the precision of the 3D reconstruction was carried out, thanks to the redundancy of data acquired to generate the model. In particular, the estimated precision of the 3D model amounts to few hundredths of millimeters, in agreement with the claimed nominal standard deviation.

Finally, the freely available software CuneiformAnalyser was successfully tested, with the aim of improving the analysis of the

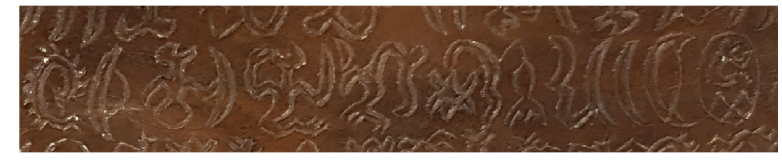

(a)

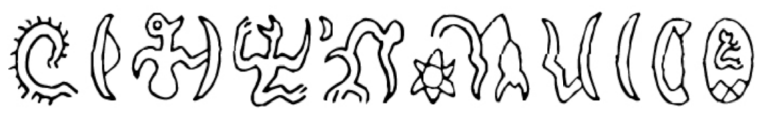

(b)

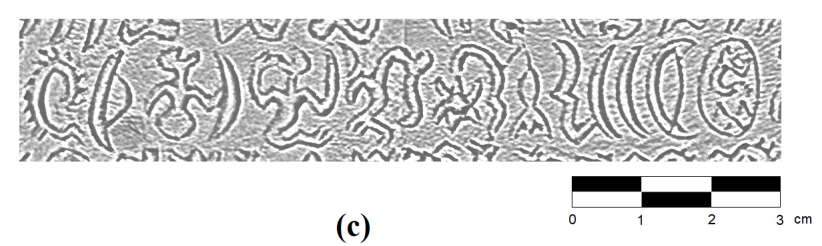

(c)

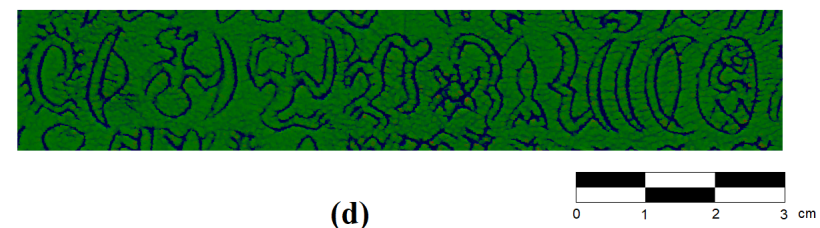

Figure 7: The Mamari tablet, side a, line 7, from glyph 19 (059) to glyph 33 (152): (a) color image; (b) normalized drawing from (Barthel, 1958); (c) 3D model rendered through CuneiformAnalyser - Radiance Scaling; (d) 3D model rendered through CuneiformAnalyser - Max Curvature

features of interest of the 3D model: indeed, the Radiance Scaling shader and the curvature analysis tools managed to effectively enhance the legibility of the signs. Indeed, the use of CuneiformAnalyser (see Figure 7(d)) might in the future assist the creation of improved line-art drawings of the Rongorongo inscriptions, and thus be conducive to further progress.

It follows that the main results achieved, as presented here, i.e. the 3D reconstruction of the Mamari tablet, will be of the utmost importance for correcting the extant editions of the Rongorongo texts, thanks to the high geometric precision it can provide. 
Moreover, the methodology described here will be further finetuned, according to the specific peculiarities and characteristics of the other objects of the Rongorongo corpus, which vary in terms of shape, dimensions and also material, since they are made of different types of wood (Barthel, 1958).

Finally, we aim to reconstruct the texture of the Mamari tablet, applying the photogrammetric technique in conjunction with the use of circular polarized filters, able to reduce the specular reflections on its shiny surface (Nicolae et al., 2014). In this way, a 3D model that includes both texture (from photogrammetry) and high detailed 3D geometry (from laser scanning) will be produced, following the method already described in (Lastilla et al., 2019).

\section{ACKNOWLEDGEMENTS}

The authors wish to thank the Museum Archives of the "Congregazione dei Sacri Cuori di Gesù e Maria" (Rome, Italy) for allowing the dissemination of the 3D model of the Mamari tablet.

The authors are also grateful to D. Fisseler, J. Günnewig, T. Harweg, G.G.W. Müller and M. Cammarosano for making the software CuneiformAnalyser freely available.

The INSCRIBE project has received funding from the European Research Council (ERC) under the European Union's Horizon 2020 research and innovation programme (grant agreement No. 771127).

\section{REFERENCES}

Affatato, S., Valigi, M. and Logozzo, S., 2017. Wear distribution detection of knee joint prostheses by means of 3D optical scanners. Materials 10(4), pp. 364. doi:10.3390/ma10040364.

Barthel, T., 1958. Grundlagen zur Entzifferung der Osterinselschrift. Walter de Gruyter.

Fischer, S. R., 1997. Rongorongo: the Easter Island script: History, Traditions, Texts. Clarendon Press.

Fisseler, D., Weichert, F., Müller, G. and Cammarosano, M., 2013. Towards an interactive and automated script feature analysis of 3D scanned cuneiform tablets. Scientific Computing and Cultural Heritage p. 16.

Girardeau-Montaut, D., 2019. Cloud Compare - 3D Point Cloud and Mesh Processing Software - Version 2.10.2. http://www . danielgm. net/cc/. Online; accessed October, 2019.

Guy, J., 1990. The lunar calendar of tablet Mamari. Journal de la Société des Océanistes 91(2), pp. 135-149.

INSCRIBE, 2018. https://site.unibo.it/inscribe/en.

INSCRIBE, 2019. 3D model of the Mamari tablet or Text C of Rongorongo script. https : //www . youtube. com/watch?v= 2RUdSmeXe4E. Online; created October, 2019.

Lastilla, L., Ravanelli, R. and Ferrara, S., 2019. 3D highquality modeling of small and complex archaeological inscribed objects: Relevant issues and proposed methodology. The International Archives of the Photogrammetry, Remote Sensing and Spatial Information Sciences XLII-2/W11, pp. 699-706. doi: 10.5194/isprs-archives-XLII-2-W11-699-2019.

Nicolae, C., Nocerino, E., Menna, F. and Remondino, F., 2014. Photogrammetry applied to problematic artefacts. The International Archives of the Photogrammetry, Remote Sensing and Spatial Information Sciences XL-5, pp. 451-456. doi: 10.5194/isprsarchives-XL-5-451-2014.
Orliac, C., 2005. The "Rongorongo" tablets from Easter Island: botanical identification and $14 \mathrm{C}$ dating. Archaeology in Oceania 40(3), pp. 115-119.

Spaelti, P., 2005-2019. Rongorongo corpus. http:// kohaumotu.org/rongorongo_org/corpus/1.html. Online; accessed October, 2019.

Vergne, R., Pacanowski, R., Barla, P., Granier, X. and Schlick, C., 2010. Radiance scaling for versatile surface enhancement. In: Proceedings of the 2010 ACM SIGGRAPH symposium on Interactive 3D Graphics and Games, ACM, pp. 143-150. doi: 10.1145/1730804.1730827. 